\title{
BEAM ELEMENT FOR LARGE DISPLACEMENT ANALYSIS OF ELASTO-PLASTIC FRAMES
}

\author{
NGUYen Dinh Kien ${ }^{1}$ And Do Quoc Quang ${ }^{2}$ \\ 1 Institute of Mechanics \\ Vietnamese Academy of Science and Technology \\ ${ }^{2}$ Research Institute of Technology for Machinery, Ministry of Industry
}

\begin{abstract}
The present paper develops a non-linear beam element for analysis of elastoplastic frames under large displacements. The finite element formulations are derived by using the co-rotational approach and expression of the virtual work. The Gauss quadrature is employed for numerically computing the element tangent stiffness matrix and internal force vector. A bilinear stress-strain relationship with isotropic hardening is adopted to update the stress. The arc-length technique based on the Newton-Raphson iterative method is employed to compute the equilibrium paths. A number of numerical examples is employed to assess the performance of the developed element. The effects of plastic action on the large displacement behavior of the structures as well as the expansion of plastic zones in the loading process are discussed.
\end{abstract}

\section{Introduction}

In the previous work $[1,2]$, the authors investigated some beam elements for assessing the behavior of elastic frames under large displacements. The finite element formulations in the work have been developed by using the co-rotational approach, in which an element attached coordinate system which continuously rotates and moves during the element deformation process was employed. The approach allowed to derive the finite element formulations in a local system, and then transfer them to a global one with the aid of the transformation matrices. In addition, the stress-strain relationship was assumed to be linear, and this assumption enabled to derive the element formulations from the expression of strain energy. As consequences, the explicit forms of the element tangent stiffness matrix and internal force vector have been obtained.

The present work aims to develop a beam element for large displacement analysis of elasto-plastic frames by lifting the restriction of linearly elastic assumption in [1,2], and the behavior of the structural material is now supposed to be elasto-plastic. The co-rotational approach is still adopted herewith, so that the work will mainly focuss on the derivation of formulations in the local system. The extension of the finite element formulations from the elastic case to the elasto-plastic one is, however, not straightforward and not simple either. With the involvement of plasticity, the structure became a dissipate system [3], and one could not able to derive the expression of strain energy. As a result, the principle of stationary potential energy, a powerful tool for elastic systems, is not able to apply for constructing the element formulations. Furthermore, the numerical integration is often 
needed to compute the tangent stiffness matrix and internal force vector, since these quantities are hardly derived in explicit forms for the plastic case. As a consequence, the computation of any real structure involving plasticity is very computer time consuming.

Taking elasto-plastic law of the material behavior into account, the derivation of finite element formulations for large displacement analysis of frames is rather a complicated task, since both the geometrically and materially nonlinearities should be considered simultaneously. The number of related elements found by the authors in the literature is quite limited. An early element, a hybrid type, was formulated by Bäcklund [4], in which the element stiffness matrix was formulated from the inversion of a flexibility matrix. In [5], Cichón constructed a beam element in the context of total Lagrangian formulation, which requires higher-order terms for assessing the new element length. In [6], Hsiao and co-workers also employed a 'body-attached' coordinate system for developing element, but the method is far from the co-rotational approach initiated by Belytschko et al. [7, 8], due to their way of choosing the local coordinates. As a result, the method by Hsiao and co-workers lent the local tangent stiffness matrix and internal force vector with the same sizes as the global ones.

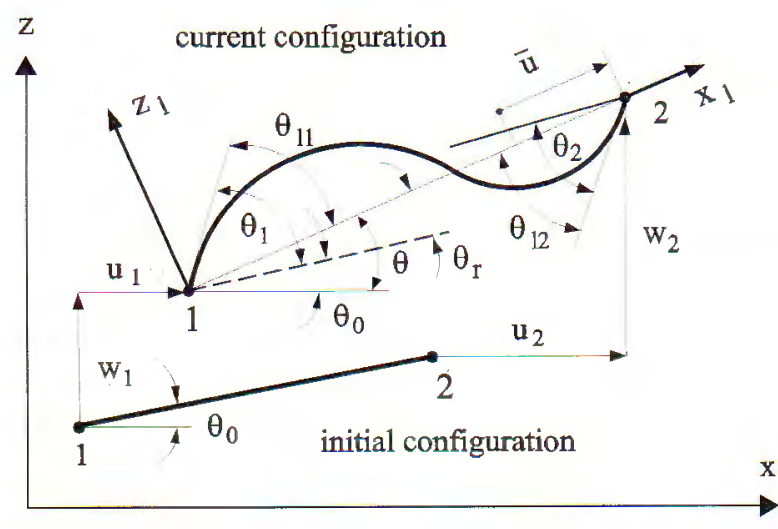

Fig. 1. Element configurations and kinematics

In [8], Belytschko et al. presented a co-rotational rod element for dynamic analysis of frame structures. In the same context of co-rotational approach in [8], Crisfield formulated a beam element based on linear definition of the local strain for instability analysis [9]. Both the work discussed in $[8,9]$ employed a local coordinate system, which continuously moves and rotates with the element during the deformation process as above mentioned. As a result, the local formulations are simple, and the size of these formulations is much smaller than that of the global one. The material behavior in both the work in $[8,9]$ is, however, assumed linearly elastic.

The present paper adopted the co-rotational approach discussed in $[8,9]$ to develop a nonlinear beam element for large displacement analysis of elasto-plastic frames. Thus, the main difference with the work in $[8,9]$ lies on derivation of the local formulations, which is presented below in Section 4. These local formulations, formulating from a bilinear elastoplastic behavior, cannot be derived explicitly, and thus require the numerical integrations. 
The involvement of the plastic deformation makes the element formulations to be complex, and the analysis is much more expensive. Besides that, a direct approach for constructing the relationship between the local and global nodal displacements, presented in Section 2, is also different from the approach discussed in [9], where a geometric method has been adopted.

\section{Local and global relationships}

The formulations needed for transferring the local formulations to the global ones in the elastic case can be derived with the aid of strain energy expression [1,2], but this approach, as discussed above, is not proper for a dissipative system of present consideration of the elasto-plastic structure. The formulations, therefore will be derived in this section by using expression of the virtual work, instead of the strain energy expression. Fig. 1 shows the configurations of a beam element in the initial and current deformed states. The element consists of two nodes, and in respective to the global system $(x, z)$, there are three nodal degrees of freedom (d.o.f) at each node: axial and transversal displacements and a rotation. The element attached coordinates $\left(x_{l}, z_{l}\right)$ (also called the local system below) are chosen as well as its original is always placed at node 1 , and the $x_{l}$ axis is directed to node 2 . With this definition, the axial displacement at node 1 and both transversal displacements in the local system are vanished. Accordingly, vectors of the element nodal displacements in the systems are respectively given by

$$
\begin{aligned}
\mathbf{d}_{l} & =\left\{\begin{array}{llll}
\bar{u} & \theta_{l 1} & \theta_{l 2}
\end{array}\right\}^{T}, \\
\mathbf{d} & =\left\{\begin{array}{llllll}
u_{1} & w_{1} & \theta_{1} & u_{2} & w_{2} & \theta_{2}
\end{array}\right\}^{T},
\end{aligned}
$$

where and later, the subscript $l$ stands for 'local'; $(\ldots)^{T}$ denotes the transpose of a vector or a matrix; $\bar{u}$ is the local axial displacement of node 2 . The local d.o.fs are related to the global ones by (see Fig. 1)

$$
\bar{u}=l_{n}-l_{o} \quad, \quad \theta_{l 1}=\theta_{1}-\theta_{r} \quad, \quad \theta_{l 2}=\theta_{2}-\theta_{r},
$$

where $l_{o}, l_{n}$ are the initial and current lengths of the element

$$
\begin{aligned}
& l_{o}=\sqrt{\left(x_{2}-x_{1}\right)^{2}+\left(z_{2}-z_{1}\right)^{2}}, \\
& l_{n}=\sqrt{\left(x_{2}+u_{2}-x_{1}-u_{1}\right)^{2}+\left(z_{2}+w_{2}-z_{1}-w_{1}\right)^{2}},
\end{aligned}
$$

and $\theta_{r}$ is the rigid rotation, which can be computed from geometry consideration as

$$
\sin \theta_{r}=s c_{o}-c s_{o} \Longrightarrow \theta_{r}=\arcsin \left(s c_{o}-c s_{o}\right),
$$

with

$$
\begin{aligned}
& c_{o}=\cos \theta_{o}=\frac{x_{2}-x_{1}}{l_{0}}, \quad c=\cos \theta=\frac{x_{2}+u_{2}-x_{1}-u_{1}}{l_{n}}, \\
& s_{0}=\sin \theta_{0}=\frac{z_{2}-z_{1}}{l_{o}}, \quad s=\sin \theta=\frac{z_{2}+w_{2}-z_{1}-w_{1}}{l_{n}},
\end{aligned}
$$


where $\theta_{0}, \theta$ are the initial and current inclined angles of the $x_{l}$ axis. Eq. (2.6) is prevailed with $\theta_{r} \leq|\pi / 2|$, but by computing $\cos \theta_{r}$, the extension to the range of $2 \pi$ is straightforward.

From Eqs. (2.4)-(2.8), the local virtual d.o.fs can be computed as

$$
\begin{aligned}
\delta \bar{u} & =c\left(\delta u_{2}-\delta u_{1}\right)+s\left(\delta w_{2}-\delta w_{1}\right)=\left[\begin{array}{lllll}
-c & -s & 0 & c & s
\end{array}\right] \delta \mathbf{d} \\
\delta \theta_{l 1} & =\delta \theta_{1}-\delta \theta_{r}=\delta \theta_{1}-\delta \theta \\
\delta \theta_{l 2} & =\delta \theta_{2}-\delta \theta_{r}=\delta \theta_{2}-\delta \theta
\end{aligned}
$$

where $\delta \mathbf{d}=\left\{\begin{array}{llllll}\delta u_{1} & \delta w_{1} & \delta \theta_{1} & \delta u_{2} & \delta w_{2} & \delta \theta_{2}\end{array}\right\}^{T}$ is the vector of virtual global d.o.fs. The far rigkthand sides of Eqs. (2.10) and (2.11) have been written with notice that $\delta \theta_{0}=0$. By differentiating $\sin \theta$, as defined in (2.8), one can obtain the expression for $\delta \theta$ as

$$
\delta \theta=\frac{\left(\delta w_{2}-\delta w_{1}\right)-s c\left(\delta u_{2}-\delta u_{1}\right)-s^{2}\left(\delta w_{2}-\delta w_{1}\right)}{c l_{n}} .
$$

With some calculations, Eq. (2.12) can be written in the form

$$
\delta \theta=\frac{1}{l_{n}}\left[\begin{array}{llllll}
s & -c & 0 & -s & c & 0
\end{array}\right] .
$$

From (2.9)-(2.11) and (2.13) we get

$$
\delta \mathbf{d}_{l}=\mathbf{B} \delta \mathbf{d}
$$

with the transformation matrix $\mathbf{B}$ given by

$$
\mathbf{B}=\frac{1}{l_{n}}\left[\begin{array}{cccccc}
-c l_{n} & -s l_{n} & 0 & c l_{n} & s l_{n} & 0 \\
-s & c & l_{n} & s & -c & 0 \\
-s & c & 0 & s & -c & l_{n}
\end{array}\right]
$$

where $c$ and $s$ are given by Eqs. (2.7) and (2.8), respectively.

Having the transformation matrix $\mathbf{B}$ derived, we can now relate the local and global formulations by equating the virtual work in both the coordinate systems as

$$
\mathcal{V}=\delta \mathbf{d}^{T} \mathbf{f}=\delta \mathbf{d}_{l}^{T} \mathbf{f}_{l}=\delta \mathbf{d}^{T} \mathbf{B}^{T} \mathbf{f}_{l},
$$

where $\mathbf{f}_{l}=\left\{\begin{array}{lllllll}N_{l} & M_{l 1} & M_{l 2}\end{array}\right\}^{T}$ and $\mathbf{f}=\left\{\begin{array}{llllll}N_{1} & Q_{1} & M_{1} & N_{2} & Q_{2} & M_{2}\end{array}\right\}^{T}$ are the vectors of local and global internal forces, respectively. From (2.16), the global internal force vector is given by

$$
\mathbf{f}=\mathbf{B}^{T} \mathbf{f}_{l} .
$$

The element tangent stiffness in the global system $\mathbf{k}_{t}$ can be obtained from differentiation of the global internal force vector $\mathbf{f}$, Eq.(2.17), as

$$
\delta \mathbf{f}=\mathbf{k}_{t} \delta \mathbf{d}=\mathbf{B}^{T} \delta \mathbf{f}_{l}+N_{l} \delta \mathbf{B}_{1}+M_{l 1} \delta \mathbf{B}_{2}+M_{l 2} \delta \mathbf{B}_{3},
$$


where $\mathbf{B}_{i},(i=1 \ldots 3)$ is the $i^{\text {th }}$ column of the matrix $\mathbf{B}^{T}$. From (2.15) one can get

$$
\delta \mathbf{B}_{1}=\frac{\mathbf{z z}^{T}}{l_{n}} \delta \mathbf{d}, \quad \delta \mathbf{B}_{2}=\delta \mathbf{B}_{3}=\frac{\left(\mathbf{r} \mathbf{z}^{T}+\mathbf{z r}^{T}\right)}{l_{n}^{2}} \delta \mathbf{d},
$$

where $\mathbf{r}$ and $\mathbf{z}$ denote the vectors with following components

$$
\begin{aligned}
& \mathbf{r}=\left[\begin{array}{llllll}
-c & -s & 0 & c & s & 0
\end{array}\right]^{T}, \\
& \mathbf{z}=\left[\begin{array}{llllll}
s-c & 0 & -s & c & 0
\end{array}\right]^{T} .
\end{aligned}
$$

From Eqs. (2.18)-(2.21), the expression for the tangent stiffness matrix $\mathbf{k}_{t}$ can be written as

$$
\underset{(6 \times 6)}{\mathbf{k}_{t}}=\mathbf{B}^{T} \underset{(3 \times 3)}{\mathbf{k}_{t l}} \mathbf{B}+\frac{\mathbf{z z}^{T}}{l_{n}} N_{l}+\frac{\left(\mathbf{r z}^{T}+\mathbf{z r}^{T}\right)}{l_{n}^{2}}\left(M_{l 1}+M_{l 2}\right),
$$

with $\mathbf{k}_{t l}=\partial \mathbf{f}_{l} / \partial \mathbf{d}_{l}$ is the local element tangent stiffness matrix.

A combination of Eqs. (2.17), (2.22) with Eqs. (2.15), (2.20) and (2.21) completely defines the global internal force vector and tangent stiffness matrix through their counterparts, and the remaining work for obtaining the element formulations is to formulate the local internal force vector $\mathbf{f}_{l}$ and the tangent stiffness matrix $\mathbf{k}_{l}$, and this work will be presented in Section 4 below.

\section{Elasto-plastic law}

A bilinear stress-strain model with isotropic hardening rule for the material as shown in Fig. 2.a is adopted in the present work. The model is represented by the so-called 'elasto-plastic' modulus $\tilde{E}$, which defined as

$$
\tilde{E}=\frac{\partial \sigma}{\partial \epsilon}=\left\{\begin{array}{l}
E \text { if }|\sigma|<\sigma_{Y} \text { or unloading } \\
E_{t} \text { if }|\sigma| \geq \sigma_{Y},
\end{array}\right.
$$

where $E, E_{t}$ are the elastic and tangent modulus, respectively; $\sigma_{Y}$ is the current yield stress with initial value $\sigma_{0}$, which can be determined from a simple tensile test. For the reverse loading path in Fig. 2.a, the yielding occurs when the stress value reaches $\sigma_{B}$-the stress at unloading point $B$. The kinematic hardening rule, which takes Bauschinger effect into consideration [10], is also widely employed in the field of structural analysis, but the present work does not investigate the effects of different hardening rules.

Suppose yielding has already occurred, then an strain increment $d \varepsilon$, from point $B$ to point $C$ in Fig. 2.b can be regarded as composed of elastic and plastic parts: $d \varepsilon=d \varepsilon_{p}+d \varepsilon_{e}$. The corresponding stress increment $d \sigma$ can be computed in various ways as (see Fig. 2.b)

$$
d \sigma=E\left(d \epsilon-d \epsilon_{p}\right), \quad d \sigma=E_{t} d \epsilon, \quad d \sigma=H d \epsilon_{p},
$$

where $H$ is the strain hardening parameter, which related to $E$ and $E_{t}$ by

$$
H=\frac{E_{t}}{1-\left(E_{t} / E\right)} \text { or } \quad E_{t}=E\left(1-\frac{E}{E+H}\right) \text {. }
$$


If $E$ is finite, and $E_{t}=0$, then $H=0$, and the material is 'elastic-perfectly' plastic [10].

If the material makes a transition from elastic to plastic, such as from point $A$ to point $B$ in Fig. 2.b, the stress increment is computed by calculating the 'elastic' fraction $m$ as'

$$
m=\frac{\sigma_{0}-\left|\sigma_{A}\right|}{\left|\sigma_{B e}\right|-\left|\sigma_{A}\right|} \quad \text { so that } \quad d \sigma=\left[m E+(1-m) E_{t}\right] d \varepsilon,
$$

where $\sigma_{A}$ is the stress at point $A ; \sigma_{B e}$ is the 'elastic' stress at point $B$, computed by assuming the total stress increment is elastic; $d \varepsilon=\varepsilon_{B}-\varepsilon_{A}$ is the total strain increment. The absolute values of the stress are needed for the case of compressive stress.
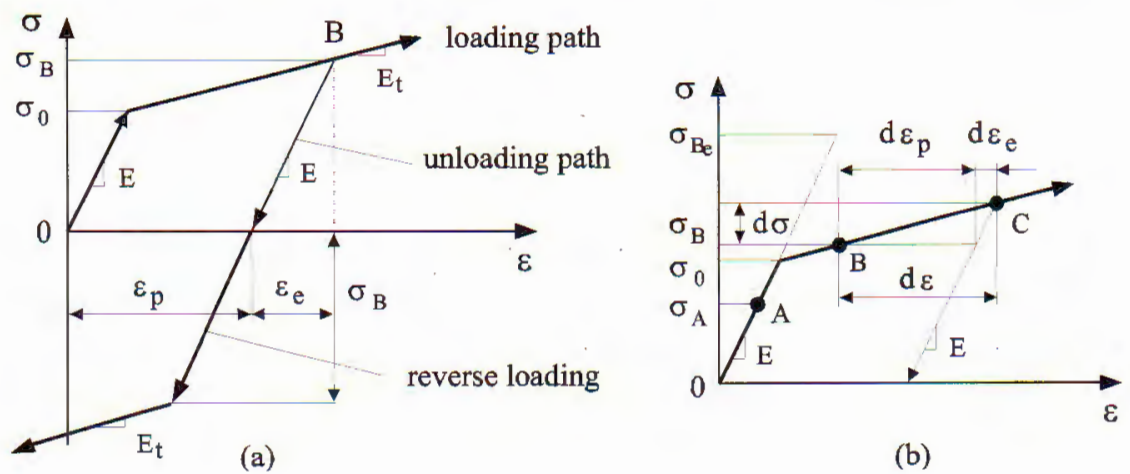

(b)

Fig. 2. Bilinear elasto-plastic model (a), and a stress increment (b)

\section{Local formulations}

This section formulates the local internal force vector and tangent stiffness matrix. To this end, a definition for local strain should be adopted, and in accordance with the previous work in [2], the shallow arch theory is employed herewith

$$
\varepsilon_{x l}=\varepsilon_{e f}+z_{l} \varkappa_{l}=\frac{1}{l_{0}} \int_{0}^{l_{0}}\left[\frac{\partial u_{l}}{\partial x_{l}}+\frac{1}{2}\left(\frac{\partial w_{l}}{\partial x_{l}}\right)^{2}\right] d x_{l}-z_{l} \frac{\partial^{2} w_{l}}{\partial x_{l}^{2}},
$$

where $\varkappa_{l}$ is the local curvature; $\varepsilon_{e f}$ is the effective strain, as explained in [2], employed in order to avoid the locking problems [9, 11]. To express the strain in Eq. (4.1) in terms of the nodal displacements defined by Eq. (2.1), the usual interpolation schemes, a linear function for $u_{l}$ and the Hermitian polynomials for $w_{l}$ are adopted as

$$
u_{l}=\frac{\xi}{l_{o}} \bar{u} \quad ; \quad w_{l}=\left\{\begin{array}{l}
\left(\xi^{2}-1\right)(\xi-1) \\
\left(\xi^{2}-1\right)(\xi+1)
\end{array}\right\}^{T}\left\{\begin{array}{l}
\theta_{l 1} \\
\theta_{l 2}
\end{array}\right\}
$$

where $\xi=\left(2 x_{l} / l_{o}-1\right),-1 \leq \xi \leq 1$, is the natural coordinate, which introduced in regard of the later development on numerical integration. Substitute (4.2) into (4.1) and notice that $\frac{\partial}{\partial x_{l}}=\frac{2}{l_{o}} \frac{\partial}{\partial \xi}$, one can easily get the expression for the local strain, which is now, for the sake of simplicity, denoted by $\varepsilon$

$$
\varepsilon=\frac{1}{l_{o}} \bar{u}+\frac{1}{15}\left(\theta_{l 1}^{2}-\frac{1}{2} \theta_{l 1} \theta_{l 2}+\theta_{l 2}^{2}\right)+\frac{z_{l}}{l_{o}}\left[(1-3 \xi) \theta_{l 1}-(1+3 \xi) \theta_{l 2}\right] .
$$


The element virtual work in the local system can be written as

$$
\mathcal{V}=\int_{V} \sigma \delta \varepsilon d V=N_{l} \delta \bar{u}+M_{l 1} \delta \theta_{l 1}+M_{l 2} \delta \theta_{l 2},
$$

where $V$ is the element volume; $d V=d A d x_{l}=\left(l_{o} / 2\right) d A d \xi$, with $A$ is the cross-sectional area of the beam; $\sigma$ is written in place of $\sigma_{x l}$. From (4.3), the virtual strain $\delta \epsilon$ can be easily computed as

$$
\begin{aligned}
\delta \epsilon= & \frac{1}{l_{o}} \delta \bar{u}+\left(\frac{2}{15} \theta_{l 1}-\frac{1}{30} \theta_{l 2}\right) \delta \theta_{l 1}+\left(\frac{2}{15} \theta_{l 2}-\frac{1}{30} \theta_{l 1}\right) \delta \theta_{l 2} \\
& +\frac{z_{l}}{l_{o}}\left[(1-3 \xi) \delta \theta_{l 1}-(1+3 \xi) \delta \theta_{l 2}\right] .
\end{aligned}
$$

Substitute (4.5) into (4.4), the local internal forces are obtained as

$$
\begin{aligned}
N_{l} & =\frac{1}{2} \int_{-1}^{1} \int_{A} \sigma d A d \xi \\
M_{l 1} & =\frac{l_{o}}{2}\left(\frac{2}{15} \theta_{l 1}-\frac{1}{30} \theta_{l 2}\right) \int_{-1}^{1} \int_{A} \sigma d A d \xi+\frac{1}{2} \int_{-1}^{1} \int_{A} z_{l}(1-3 \xi) \sigma d A d \xi \\
M_{l 2} & =\frac{l_{o}}{2}\left(\frac{2}{15} \theta_{l 2}-\frac{1}{30} \theta_{l 1}\right) \int_{-1}^{1} \int_{A} \sigma d A d \xi-\frac{1}{2} \int_{-1}^{1} \int_{A} z_{l}(1+3 \xi) \sigma d A d \xi
\end{aligned}
$$

Having the local internal forces computed, the coefficients for the local tangent stiffness matrix $\mathbf{k}_{t l}$ are obtained from differentiation of $\mathbf{f}_{l}$, with respective to the local d.o.fs. Noticing $\delta \sigma=\tilde{E} \delta \epsilon$, with $\delta \epsilon$ given by Eq. (4.5), we can get

$$
\begin{aligned}
k_{t l}(1,1) & =\frac{1}{2 l_{o}} \int_{-1}^{1} \int_{A} \tilde{E} d A d \xi \\
k_{t l}(1,2) & =\frac{1}{2}\left(\frac{2}{15} \theta_{l 1}-\frac{1}{30} \theta_{l 2}\right) \int_{-1}^{1} \int_{A} \tilde{E} d A d \xi+\frac{1}{2 l_{o}} \int_{-1}^{1} \int_{A} z_{l}(1-3 \xi) \tilde{E} d A d \xi \\
k_{t l}(1,3) & =\frac{1}{2}\left(\frac{2}{15} \theta_{l 2}-\frac{1}{30} \theta_{l 1}\right) \int_{-1}^{1} \int_{A} \tilde{E} d A d \xi-\frac{1}{2 l_{o}} \int_{-1}^{1} \int_{A} z_{l}(1+3 \xi) \tilde{E} d A d \xi \\
k_{t l}(2,2) & =\frac{l_{o}}{15} \int_{-1}^{1} \int_{A} \sigma d A d \xi+\frac{l_{o}}{2}\left(\frac{2}{15} \theta_{l 1}-\frac{1}{30} \theta_{l 2}\right)^{2} \int_{-1}^{1} \int_{A} \tilde{E} d A d \xi \\
& +\left(\frac{2}{15} \theta_{l 1}-\frac{1}{30} \theta_{l 2}\right) \int_{-1}^{1} \int_{A} z_{l}(1-3 \xi) \tilde{E} d A d \xi+\frac{1}{2 l_{o}} \int_{-1}^{1} \int_{A} z_{l}^{2}(1-3 \xi)^{2} \tilde{E} d A d \xi \\
k_{t l}(2,3) & =\frac{l_{o}}{2}\left(\frac{2}{15} \theta_{l 1}-\frac{1}{30} \theta_{l 2}\right)\left(\frac{2}{15} \theta_{l 2}-\frac{1}{30} \theta_{l 1}\right) \int_{-1}^{1} \int_{A} \tilde{E} d A d \xi \\
& -\frac{l_{o}}{60} \int_{-1}^{1} \int_{A} \sigma d A d \xi-\frac{1}{2}\left(\frac{2}{15} \theta_{l 1}-\frac{1}{30} \theta_{l 2}\right) \int_{-1}^{1} \int_{A} z_{l}(1+3 \xi) \tilde{E} d A d \xi \\
& +\frac{1}{2}\left(\frac{2}{15} \theta_{l 2}-\frac{1}{30} \theta_{l 1}\right) \int_{-1}^{1} \int_{A} z_{l}(1-3 \xi) \tilde{E} d A d \xi-\frac{1}{2 l_{o}} \int_{-1}^{1} \int_{A} z_{l}^{2}\left(1-9 \xi^{2}\right) \tilde{E} d A d \xi
\end{aligned}
$$




$$
\begin{aligned}
k_{t l}(3,3) & =\frac{l_{o}}{15} \int_{-1}^{1} \int_{A} \sigma d A d \xi+\frac{l_{o}}{2}\left(\frac{2}{15} \theta_{l 2}-\frac{1}{30} \theta_{l 1}\right)^{2} \int_{-1}^{1} \int_{A} \tilde{E} d A d \xi \\
& -\left(\frac{2}{15} \theta_{l 2}-\frac{1}{30} \theta_{l 1}\right) \int_{-1}^{1} \int_{A} z_{l}(1+3 \xi) \tilde{E} d A d \xi+\frac{1}{2 l_{o}} \int_{-1}^{1} \int_{A} z_{l}^{2}(1+3 \xi)^{2} \tilde{E} d A d \xi,
\end{aligned}
$$

and $\quad k_{t l}(2,1)=k_{t l}(1,2) ; \quad k_{t l}(3,1)=k_{t l}(1,3) ; \quad k_{t l}(3,2)=k_{t l}(2,3)$.

Eqs. (4.6)-(4.14) combining with Eqs. (2.17) and (2.22) give the global internal force vector and tangent stiffness matrix, and completely define the element formulations. If the elastic behavior is assumed, $\sigma=E \varepsilon$, the integrations in (4.6)-(4.14) can be computed explicitly, and Eqs.(4.6)-(4.14) are reduced to the following explicit forms

$$
\begin{aligned}
N_{l} & =E A\left(\frac{\bar{u}}{l_{o}}+\frac{\theta_{l 1}^{2}}{15}-\frac{\theta_{l 1} \theta_{l 2}}{30}+\frac{\theta_{l 2}^{2}}{15}\right), \\
M_{l 1} & =\frac{l_{o}}{30} E A\left(4 \theta_{l 1}-\theta_{l 2}\right)\left(\frac{\bar{u}}{l_{o}}+\frac{\theta_{l 1}^{2}}{15}-\frac{\theta_{l 1} \theta_{l 2}}{30}+\frac{\theta_{l 2}^{2}}{15}\right)+\frac{2}{l_{o}} E I\left(2 \theta_{l 1}+\theta_{l 2}\right), \\
M_{l 2} & =\frac{l_{o}}{30} E A\left(-\theta_{l 1}+4 \theta_{l 2}\right)\left(\frac{\bar{u}}{l_{o}}+\frac{\theta_{L l 1}^{2}}{15}-\frac{\theta_{l 1} \theta_{l 2}}{30}+\frac{\theta_{l 2}^{2}}{15}\right)+\frac{2}{l_{o}} E I\left(\theta_{l 1}+2 \theta_{l 2}\right)
\end{aligned}
$$

and

$$
\begin{aligned}
& k_{t l}(1,1)=\frac{1}{l_{o}} E A \\
& k_{t l}(1,2)=\frac{1}{30} E A\left(4 \theta_{l 1}-\theta_{l 2}\right) \\
& k_{t l}(1,3)=\frac{1}{30} E A\left(-\theta_{l 1}+4 \theta_{l 2}\right) \\
& k_{t l}(2,2)=\frac{l_{o}}{30} E A\left[\frac{1}{30}\left(4 \theta_{l 1}-\theta_{l 2}\right)^{2}+4\left(\frac{\bar{u}}{l_{o}}+\frac{\theta_{l 1}^{2}}{15}-\frac{\theta_{l 1} \theta_{l 2}}{30}+\frac{\theta_{l 2}^{2}}{15}\right)\right]+\frac{4}{l_{o}} E I \\
& k_{t l}(3,3)=\frac{l_{o}}{30} E A\left[\frac{1}{30}\left(-\theta_{l 1}+4 \theta_{l 2}\right)^{2}+4\left(\frac{\bar{u}}{l_{o}}+\frac{\theta_{l 1}^{2}}{15}-\frac{\theta_{l 1} \theta_{l 2}}{30}+\frac{\theta_{l 2}^{2}}{15}\right)\right]+\frac{4}{l_{o}} E I \\
& k_{t l}(2,3)=\frac{l_{o}}{30} E A\left[\frac{\left(4 \theta_{l 1}-\theta_{l 2}\right)\left(4 \theta_{l 2}-\theta_{l 1}\right)}{30}-\left(\frac{\bar{u}}{l_{o}}+\frac{\theta_{l 1}^{2}}{15}-\frac{\theta_{l 1} \theta_{l 2}}{30}+\frac{\theta_{l 2}^{2}}{15}\right)\right]+\frac{2}{l_{o}} E I, \\
& k_{t l}(2,1)=k_{t l}(1,2) ; \quad k_{t l}(3,1)=k_{t l}(1,3) ; \quad k_{t l}(3,2)=k_{t l}(2,3) .
\end{aligned}
$$

It is noted herewith Eqs. (4.15)-(4.23) are previously derived in [2] for the case of shallow arch element.

\section{Computational procedures}

With involvement of the plastic deformation, the integrations in Eqs. (4.6)-(4.14) cannot be computed analytically, and the numerical integration should be employed. The Gauss quadrature with four points along the length and seven points through the depth of the 
element is adopted in the present work. Two Gauss-point quadrature along $x_{l}$ axis gives exact integration for the elastic case (since the strain energy $U=\frac{1}{2} \int_{V} E \varepsilon^{2} d V$ is a second order polynomial in $\xi$ ), but four-point Gauss quadrature is employed in order to investigate the plastic expansion along the beam length. The positions and weights for Gauss quadrature are given in [12].

The obtained element formulations are assembled into the internal force vector and tangent stiffness matrix at the structural level in order to construct the equilibrium equations, which can be written in the forms $[8,9]$

$$
\mathbf{R}(\mathbf{D}, \lambda)=\mathbf{F}_{\text {in }}-\mathbf{F}_{e x}=\mathbf{F}_{\text {in }}-\lambda \mathbf{f}_{e x}=\mathbf{0},
$$

where $\mathbf{R}$ is the out of balance force vector; $\mathbf{F}_{\text {in }}$ and $\mathbf{F}_{\text {ex }}$ are the vectors of internal and external forces, respectively; $\lambda$ is the load parameter, and $\mathbf{f}_{e x}$ is the fixed normalized external load vector. Eq. (5.1) can be solved by the incremental-iterative strategy based on Newton-Raphson method. In order to obtain the complete equilibrium paths, Eq. (5.1) is supplemented by the constrain equation to form the arc-length method as discussed in the authors' previous work [2].

To compute integrals in Eqs. (4.6)-(4.14), the values of stress and 'elasto-plastic' modulus at the Gauss point should be evaluated, and comparing to the elastic analysis, this procedure is an additional part. Because the incremental nature of plastic equations, Eqs. (3.2) and (3.4), in order to compute the new stress from a strain increment, values of the strain and stress at the Gauss points should be stored for each equilibrium point. Furthermore, since the hardening effect is taken into consideration, values of the yield stress are also required to store and update. To illustrate this computational process, a computer subroutine written in MATLAB [13], a Matlab function called eplaSE, is provided in the Appendix. The function shows how a strain increment is computed from the current global nodal d.o.f, and then the stress is updated by using Eqs. (3.1), (3.2) or (3.4). The strain hardening is taken into consideration in the function by updating the yield stress at Gauss points for each increment. The function is developed in close with the discussed theory, but it may not be optimized in the computational context.

\section{Numerical examples}

\subsection{Cantilever beam under tip load}

The cantilever beam with geometry and material data shown in Fig. 3 was proposed by Kondoh and Atluri in [14]. The original data were given in English units of measurement, but for easier imagination, they have been convected to SI units herewith. The beam is analyzed by eight equal elements, and the dependence of displacements at the free end on the external load $P$ is investigated.

Fig. 4 shows the computed equilibrium paths of the beam, which are in excellent with those obtained in [14]. The expansion of plasticity in the beam is investigated by examining the values of stress at the integration points for each increment, and the zones with plastic points in the beam at various load levels are shown in Fig. 5. From Fig. 5, one can see that the plastic zone in the tensile region is slightly larger than the corresponding zone in 
the compressive region. This result is physically reasonable, since the neutral axis of the beam is in tension, and the strain, defined by Eq. (4.3), is a combination of the positive membrane strain and the bending strain. The computed values of stress and strain at the three sampling points (Gauss points) in Fig. 5, given in Table. 1, further confirm this fact.

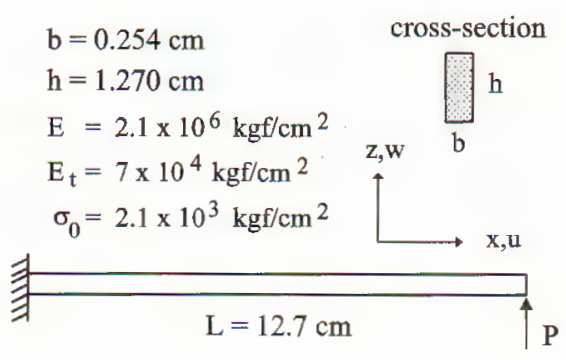

Fig. 3. Cantilever beam under tip load: geometry and material data (ref. [14])

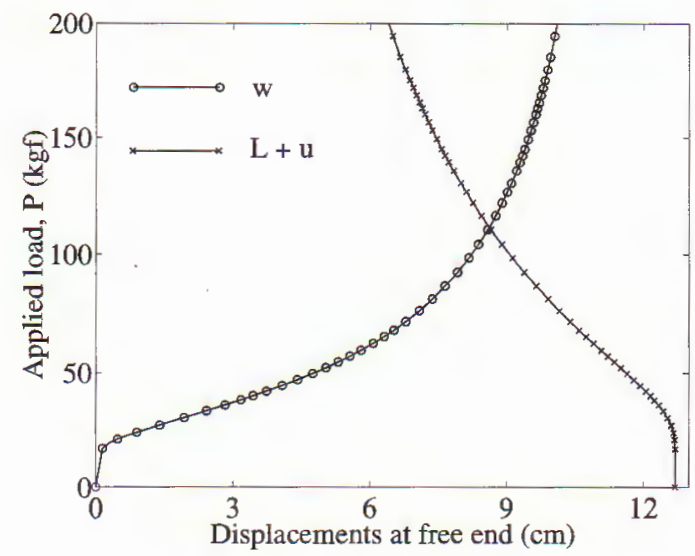

Fig. 4. Cantilever beam: applied load versus displacements at free end

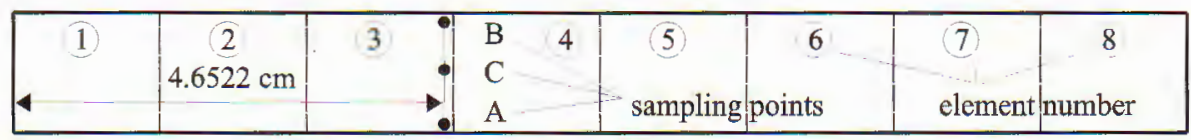
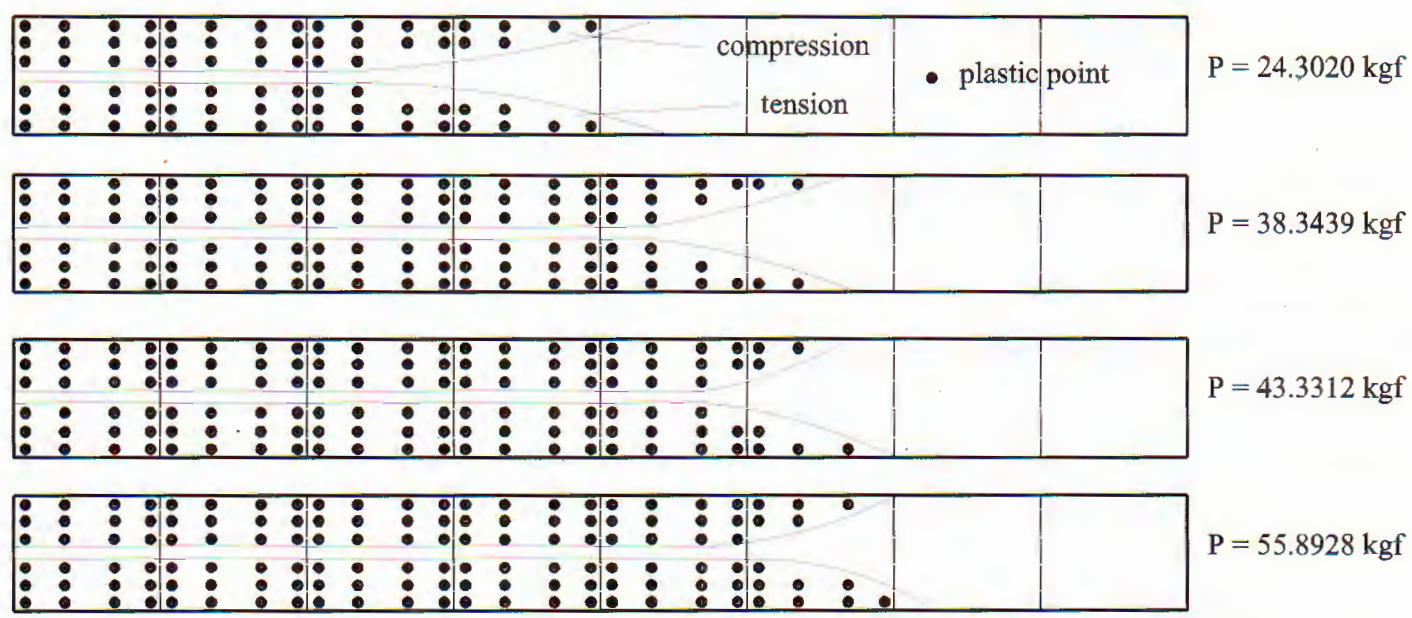

Fig. 5. Cantilever beam: position of sampling points and plastic zones 
Table 1. Cantilever beam: values of stress and strain at three sampling points in Fig. 5

\begin{tabular}{ccccccc}
\hline $\begin{array}{c}\mathrm{P} \\
(\mathrm{kgf})\end{array}$ & $\begin{array}{c}\sigma_{A} \\
\left(10^{3} \mathrm{kgf} / \mathrm{cm}^{2}\right)\end{array}$ & $\begin{array}{c}\epsilon_{A} \\
10^{-3}\end{array}$ & $\begin{array}{c}\sigma_{B} \\
\left(10^{3} \mathrm{kgf} / \mathrm{cm}^{2}\right)\end{array}$ & $\begin{array}{c}\epsilon_{B} \\
10^{-3}\end{array}$ & $\begin{array}{c}\sigma_{C} \\
\left(10^{3} \mathrm{kgf} / \mathrm{cm}^{2}\right)\end{array}$ & $\begin{array}{c}\epsilon_{C} \\
10^{-3}\end{array}$ \\
\hline 24.3020 & 2.1425 & 1.4737 & -2.1416 & -1.4612 & 0.0132 & 0.0063 \\
32.1975 & 2.7480 & 10.0860 & -2.7431 & -10.0157 & 0.0741 & 0.0352 \\
38.3439 & 3.3114 & 18.0993 & -3.3032 & -17.9819 & 0.1239 & 0.0587 \\
43.3212 & 3.7144 & 23.8312 & -3.7031 & -23.6709 & 0.1690 & 0.0801 \\
55.8928 & 4.5429 & 35.6157 & -4.5232 & -35.3345 & 0.2965 & 0.1406 \\
\hline
\end{tabular}

\subsection{Asymmetric frame}

The asymmetric frame in Fig. 6 was firstly studied by Cichón in [5], and then by Hsiao and co-workers in [6]. The analysis is performed herewith by using ten equal elements, five for each beam. The applied load versus displacements at the loading point is shown in Fig. 7, where for the purpose of comparison, the curves computed by Cichón are also displayed.

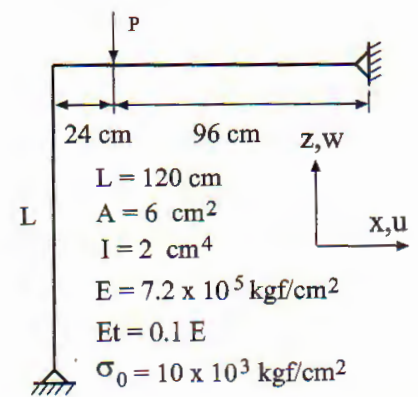

Fig. 6. Asymmetric frame under concentrate load: geometry and material data (ref. [5])

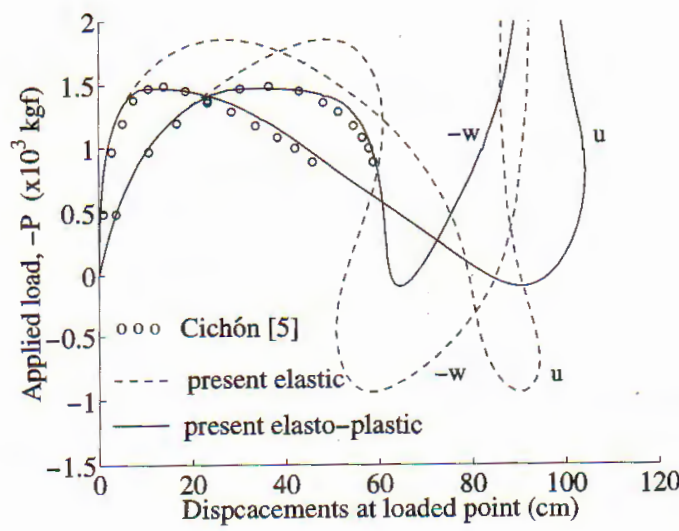

Fig. \%. Asymmetric frame: applied load versus displacements at loaded point

As seen from Fig. 7, the load-displacement curves obtained in the present study are quite agrement with those reported by Cichón using the total Lagrangian approach, up to the lateral displacement of above $60 \mathrm{~cm}$, at least. The work in [5], and in [6] either, did not show the displacement beyond $60 \mathrm{~cm}$. The large displacement behavior of the frame obtained by the elasto-plastic analysis is clearly different from that obtained by the elastic analysis. As seen from Fig. 7, by assuming the linearly elastic behavior, we have considerably overestimated the limit load of the structure. From the numerical investigation in this example, we can conclude that the effects of plasticity in some practical cases is important and it should be taken into account in the large displacement analysis of frame structures.

The authors have also employed some higher-order Gauss quadrature in analyzing this example, but the obtained results show very little difference. It is noted that both Cichón 
and Hsiao and co-workers employed Lobatto rule [9], four points along the element length and five points through the depth, in computing their internal forces and tangent stiffness matrix. The Lobatto rule has points on the beam surface which may sooner predict the plasticity, but by increasing the number of integration through the beam depth, the authors see almost no difference in the numerical results.

\subsection{Elasto-plastic buckling of Euler beam}

The elasto-plastic buckling of the simply supported beam subjected to axial forces, which often called Euler beam in the literature, is investigated in this example. The beam with length of $100 \mathrm{~cm}$, and a quare cross-section of $(5 \mathrm{~cm} \times 5 \mathrm{~cm})$, is made of the same material as the asymmetric frame in Fig. 6 . To compute the secondary buckling paths, a small transversal load, $\gamma P$ with $\gamma=0.001$, is introduced as a perturbation load. A mesh of eight equal developed elements is employed in the analysis.

Fig. 8 shows the equilibrium paths obtained by elastic and elasto-plastic analyses, where $P_{e}=-\frac{\pi^{2} E I}{L^{2}}$ is the Euler load. As noted from the figure that the behavior of the beam in the post-buckling region obtained by the elastic analysis is very different from that obtained by the elasto-plastic analysis. For the case of elastic analysis, the computed path shows the stable state in the post-buckling region, which is in agrement with the theory of the 'elastica' beam [15, 16]. The path obtained by the elasto-plastic analysis, however becomes unstable beyond point $P_{0}$ (see Fig. 8), where the material at the outer layers of the beam makes a transition from elastic to plastic.

By computing the equilibrium paths with a range of tangent modulus $E_{t}$ of 0 to $0.7 E$ as illustrated in Fig. 9, we can conclude that the position of the 'transition point' $P_{0}$ in the present example is not depended on the value of $E_{t}$, but on $\sigma_{0}$ - the initial yield stress. The paths displayed in Fig. 9 also show that the tangent modulus, that is the hardening parameter $H$ defined in Eq. (3.3), affects the post-buckling strength, measured in term of $P / P_{e}$. A higher hardening parameter is, the more post-buckling strength observers, and this result is physically reasonable.

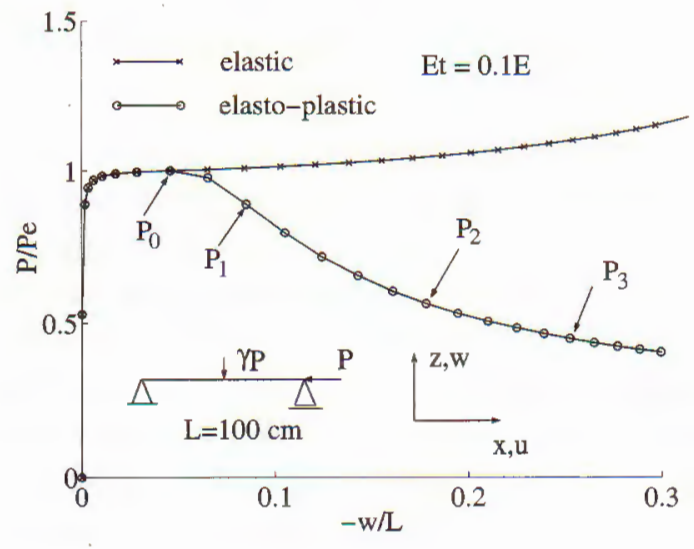

Fig. 8. Euler beam: equilibrium paths obtained by elastic and elasto-plastic analyses

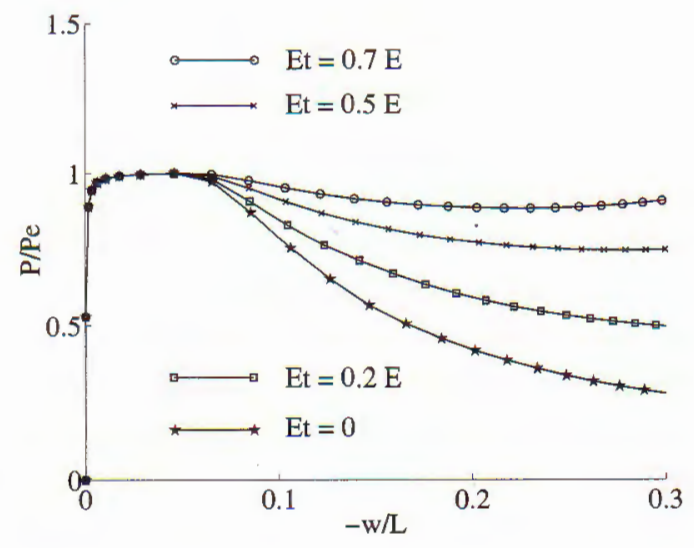

Fig. 9. Euler beam: equilibrium paths with various values of tangent modulus $E_{t}$ 
Fig. 10 shows the plastic points and their covered zones in the beam corresponding to the points $P_{1}, P_{2}$, and $P_{3}$ in Fig. 8 . In moving from point $P_{1}$ to point $P_{3}$, the plastic zones gradually expand towards the beam neutral axis from the outer layers, and to the two beam ends from the middle. In contrast to the cantilever beam in Sub-section 6.1, the plastic zone in compression in the present example is slightly larger than the corresponding zone in tension, and this fact is resulted from the negative membrane strain defined by the first two parts of Eq. (4.3). The computed values of stress and strain at the three sampling points in Fig. 10 also further confirm this result.

element number

\begin{tabular}{|c|c|c|c|c|c|c|c|c|}
\hline 1 & 2 & 3 & 4 & $\mathrm{~B}$ & 5 & 6 & 7 & 8 \\
\hline & & $49.1250 \mathrm{~cm}$ & & $\mathrm{C}$ & & & \\
\hline
\end{tabular}

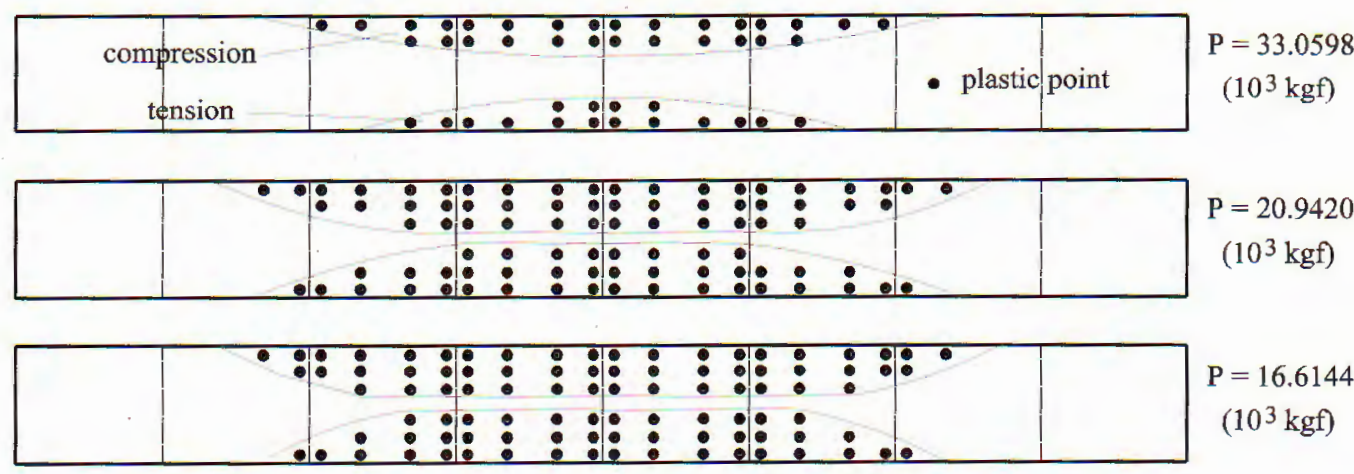

Fig. 10. Euler beam: position of sampling points and plastic zones

It is necessary to note that, in moving from point $P_{0}$ to point $P_{3}$, we are not in the unloading process as mentioned in Sec.3 and illustrated in Fig. 2.a, even the applied load $P$ (in absolute value) is in descent. The absolute values of both stress and strain, as given in Table 2, are increased, and the material is flowed plastically. That means we are still in the loading process. The decrease in the external load may be explained by the lower effective area of the cross-section, which is resulted from expansion of the plastic zones as suggested by Fung and Tong in [3], in which the plastic behavior of a bar under tensile test has been investigated.

Table 2. Euler beam: values of stress and strain at three sampling points in Fig. 10

\begin{tabular}{ccccccc}
\hline$-\mathrm{P}$ & $\sigma_{A}$ & $\varepsilon_{A}$ & $\sigma_{B}$ & $\varepsilon_{B}$ & $\sigma_{C}$ & $\varepsilon_{C}$ \\
$\left(10^{3} \mathrm{kgf}\right)$ & $\left(10^{3} \mathrm{kgf} / \mathrm{cm}^{2}\right)$ & $10^{-3}$ & $\left(10^{3} \mathrm{kgf} / \mathrm{cm}^{2}\right)$ & $10^{-3}$ & $\left(10^{3} \mathrm{kgf} / \mathrm{cm}^{2}\right)$ & $10^{-3}$ \\
\hline 33.0598 & 10.4561 & 20.2246 & -10.8615 & -25.8542 & -2.0267 & -2.8148 \\
20.9420 & 13.4701 & 62.0848 & -14.0446 & -70.0633 & -2.8723 & -3.9893 \\
16.6144 & 15.6498 & 92.3580 & -16.0989 & -98.5957 & -2.2456 & -3.1189 \\
\hline
\end{tabular}




\section{Conclusions}

A non-linear beam element for in-plane analysis of elasto-plastic frames under large displacements has been formulated in this paper. The element, taking the axial displacements into account, has been formulated by using the co-rotational approach and expression of the virtual work. The Gauss quadrature has been employed in computing the local internal force vector and the tangent stiffness matrix. The structural material has been assumed to be bilinear with isotropic hardening rule, and this law has been employed to update the stress and yield stress at the Gauss points.

The investigation from the numerical examples has shown a good performance of the developed element. It has also shown that, by lifting the linearly elastic assumption, the behavior of the frames under large displacements has been clearly changed. In particular cases, such as the structure in Sub-section 6.2, the limit load might be considerably overestimated by ignoring the plastic deformation. In the instability analysis, as shown by the example in Sub-section 6.3, with the involvement of plasticity, the nature of post-buckling behavior of the structure might completely be different. The investigation of plastic expansion has contributed to the understanding the behavior of the elasto-plastic frames under large displacements.

Acknowledgements. The authors would like to thank Prof. Ku Mo Hsiao of the National Chiao Tung University, Taiwan for useful advice in the numerical algorithm. The practical help by Mr. Duong Quang Viet, a Ph.D student at the Tokyo Institute of Technology is gratefully acknowledged.

Appendix. This Appendix lists the MATLAB commands for computing values of the new strain and stress, and updating the yield stress at Gauss points of the element. To this end, a natural coordinate, $\zeta=\frac{2}{h} z_{l}$, is introduced, so that with $z_{l} \in\left[-\frac{h}{2}, \frac{h}{2}\right] \rightarrow \zeta \in$ $[-1,1]$, where $h$ is the beam thickness. Besides the geometry and material parameters, the following input data should be included: $d$-the vector of nodal displacements, defined in Eq. (2.2); nGX, nGZ-the number of Gauss points along $x_{l}$ and $z_{l}$ axes, respectively; epso, sigo, sigYo-the vectors of strains, stresses and yield stresses at the Gauss points of the element. The function calls other function, namely ptwt2 (nGX,nGZ), in which the positions and weights for Gauss quadrature are listed [12]. The explanations, including the citations of developed formulations are provided for the sake of easily understanding.

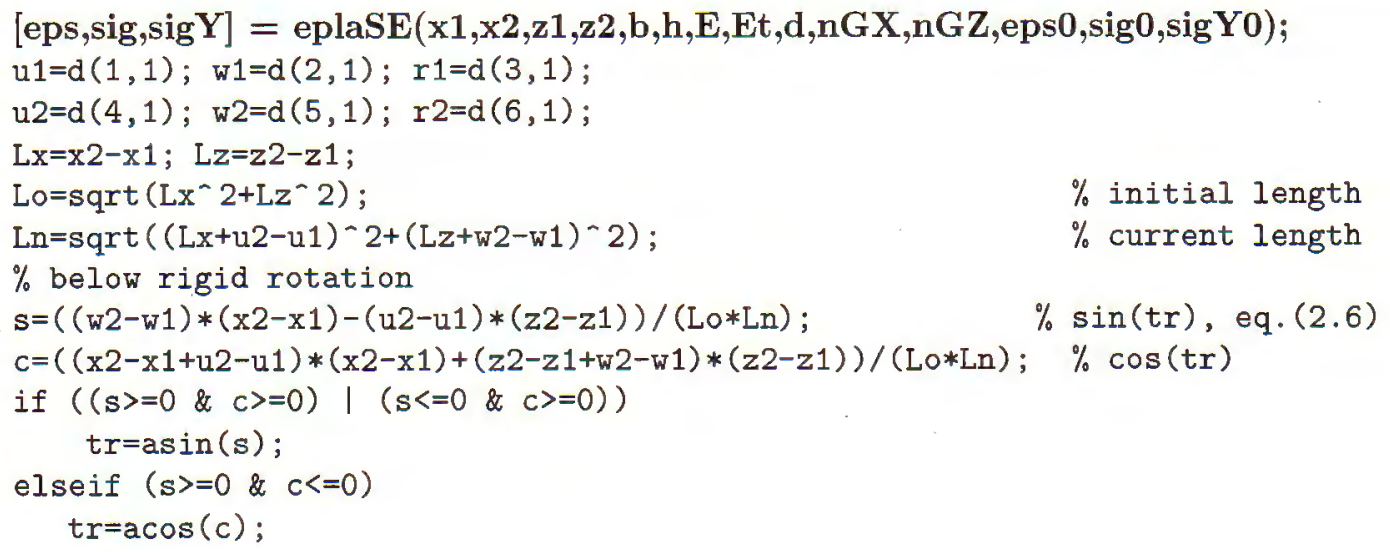




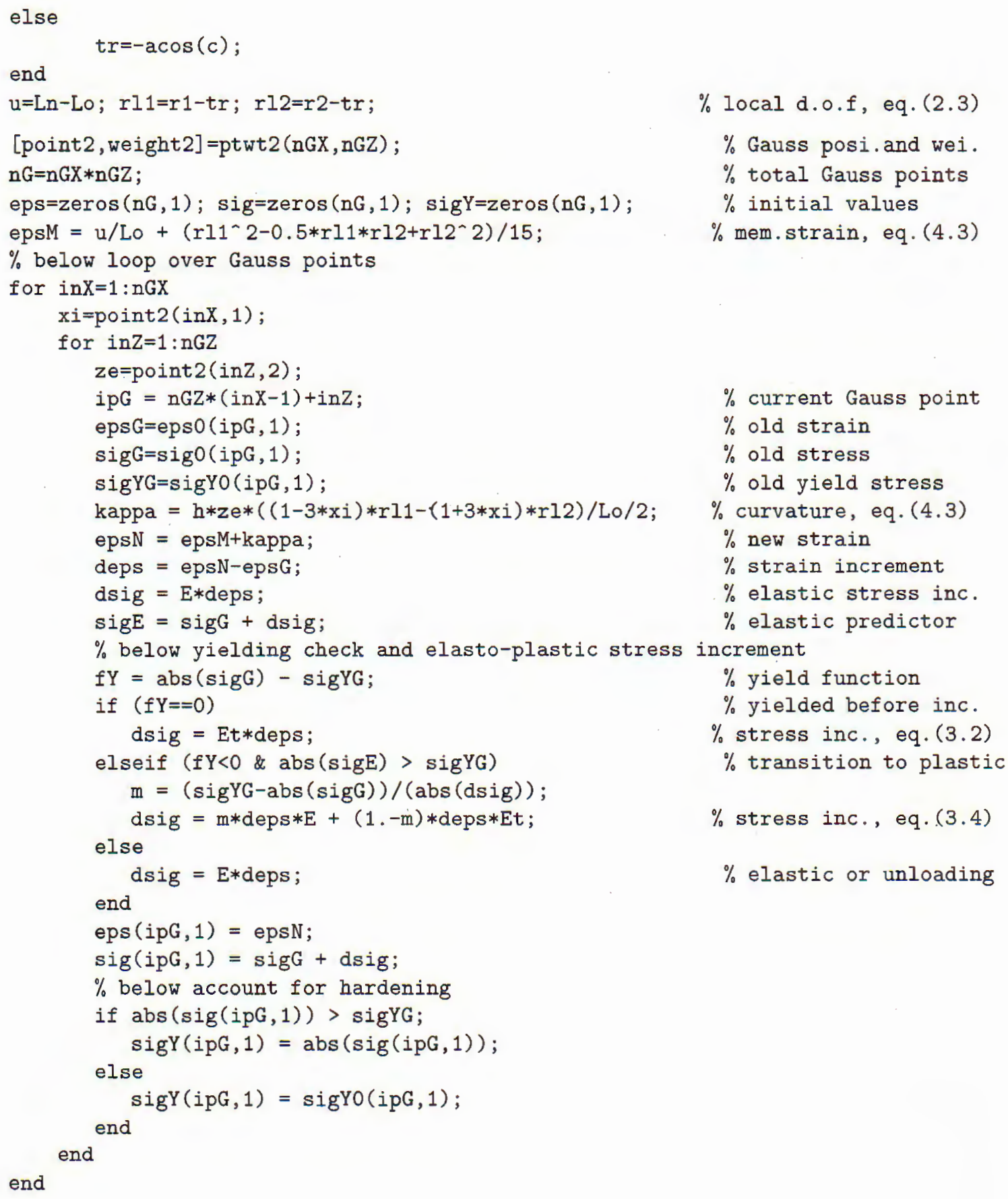

\section{References}

1. Nguyen D. K., A non-linear element for analyzing elastic frame at large defiections, Vietnam Journal of Mechanics 22 (2000) 19-28.

2. Nguyen D. K. and Do Q. Q., Large deflections analysis of frames by elements contaning higer-order terms, Vietnam Journal of Mechanics 25 (2003) 243-254. 
3. Fung Y. C. and Tong P., Classical and Computational Solid Mechanics, World scientific, Singapore, 2001.

4. Bäcklund J., Large deflection analysis of elasto-plastic beams and frames, International Journal of Mechanics Science 18 (1976) 269-277.

5. Cichón C., Large displacement in-plane analysis of elastic-plastic frames, Computer \& Structures 19 (1984) 737-745.

6. Hsiao K. M., Huo F. Y. and Spiliopoulos K. V., Large displacement analysis of elasto-plastic frames, Computer \& Structures 28 (1988) 627-633.

7. Belytschko T. and Glaum L. W., Application of higher order co-rotational stretch theories to nonlinear finite element analysis, Computer 6 Structures 10 (1979) 175-182.

8. Belytschko T., Liu W. K. and Moran B., Nonlinear Finite Elements for Continua and Structures, John Wiley \& Sons, Chichester, 2000.

9. Crisfield M. A., Non-Linear Finite Element Analysis of Solids and Structures, Volume 1: Essentials, John Wiley \& Sons, Chichester, 1991.

10. Mendenson A., Plasticity: Theory and Application, The Macmillan Co., New York, 1968.

11. Luo Y. H., Explanation and elimination of shear locking and membrane locking with field consistence approach, Computer Methods in Applied Mechanics and Engineering 162 (1998), 249-269.

12. Mathews J. H. and Fink K. D., Numerical Methods Using Matlab, Third, Prentice Hall International, New Jersey, 1999.

13. Matläb, High-Performance Numeric Computation and Visualization Software, Reference guide, The Math Works Inc., New York, 1992.

14. Kondoh K. and Atluri N., Large-deformation, elasto-plastic analysis of frames under nonconserveative loading, using explicitely derived tangent stiffness based on assumed stresses, Computational Mechanics 2 (1987) 1-25.

15. Shames I. H. and Dym C.L., Energy and Finite Element Methods in Structural Mechanics, McGraw-Hill, New York, 1985.

16. Timoshenko S. P. and Gere J. M., Theory of Elastic Stability, Second, McGraw-Hill, New York, 1961.

Received August 8, 2003

\section{PHẦ TƯ DÀM CHO PHÂN TÍCH CHUYỂN VI LỚN CƯA KHUNG ĐÀN-DÉO}

Bài báo xây dựng phần từ dầm phi tuyến cho phân tích khung đàn-déo chịu chuyển vị lớn. Công thức phần tử được thiết lập nhờ phương pháp hệ tọa độ đồng hành và biều thức công ảo. Ma trận độ cứng và véc-tơ nội lực phần tử được tính nhờ phép cầu phương Gauss. Ứng suất tại các điểm cầu phương được cập nhật trên cơ sở giả thiết quan hệ ứng suất-biến dạng tuân theo quy luật lưỡng tuyến tính với luật tái bền đằng hướng. Đường cân bằng của kết cấu được xây dựng trên cơ sở thuật toán độ dài cung và phương pháp lặp Newton-Raphson. Đối xứ cưa phần tử được đánh giá qua các ví dụ. Kết quả số cho phép đưa ra một số nhận xét về ảnh hương của chảy dẻo đến đối xử của kết cấu có chuyển vị lớn và sự lan truyền vùng dẻo trong quá trình chất tái.

e-mail: ndkien@im01.ac.vn 